\title{
A GENERIC PATTERN MATCHING APPROACH FOR MULTIPLE EVENTS
}

\author{
R.Pradeepa ${ }^{1}$, L. Sharmila \\ P.G Student, M.E CSE, Alpha College of Engineering, Chennai, T.N, India ${ }^{1}$ \\ Assistant professor, Dept. of M.E CSE, Alpha College of Engineering, Chennai, T.N, India ${ }^{2}$
}

\begin{abstract}
Data mining is a highly evolving and developing frontier in data and information systems and their applications. Its a concept of extracting data from large data set and converts into understandable format. Automated prospective analysis and knowledge driven decisions make data more proactive. Pattern matching used for control flow of patterns, checks similarity between already stored templates with input template. Patterns are procedure for converting input data. The existing system pay-as-you-go style matching technique has poor discriminative feature were this technique depend mainly on time. So it will affect $1: \mathrm{n}$ matching for events and when matching events with heterogeneous data it may result in duplication of data. In proposed system, as Generic Pattern Matching technique implies to validate similarity between two events. Number of events internally stores and maintains all the characteristics of events. Patterns are compared with all stored patterns with in the data set. Pattern matching enhances several events to improve the performance and efficiency of events. This is independent of time so $1: \mathrm{n}$ issue can be sorted out. It will also retrieve exact result without any approximate value.
\end{abstract}

Keywords: Pattern Matching, Generic Pattern Matching, Patterns, Automated prospective analysis.

\section{INTRODUCTION}

Many enterprises using data mining technique to extracting data from larger data sets and convert into the predictable format for further appliance. Data mining based on KDD (Knowledge Discovery in Databases) process is a computational process of discovering pattern, regular expression, strings, and finite automata from large data sets. Data mining automatically search from the given data set and discovers the patterns using analysis technique and also finds hidden information from datasets. This fields combines tools both from statistics and artificial intelligent with database management to analyze collection of large data sets. Data mining is process of extraction of useful patterns from data sources, e.g., databases, texts, web, image.

The theme of data mining concepts is extracting related information that corresponds to given input patterns. It is also termed as extraction of implicit, previously unknown and potentially useful information of data. The discovery and analysis by semi-automatic means of large bulk quantities of data in order to discover meaningful patterns. Data mining uses information from previous data to analyze the outcome of a particular problem or situation that may arise as a result of it. Data mining interprets its data into real time analysis and cause that can be used in business and organizations. Data mining includes initial exploration, model building, evaluation, and deployment. Data mining can be a cause issue when only for selected information, which is not representative of the overall sample group, is used to prove a certain criteria.

Matching checks is the similarity between two patterns based on score function analysis. Pattern matching implies on outputting the locations of a pattern within a token sequence to output some component of the
Matched pattern and a substitute the matching pattern with some other token sequence. Regular Expression (RE) is a set of characters that forms a meaningful pattern, mainly focus in pattern matching with strings. RE which performs search, matching and replacing patterns based on given input pattern . Regular Expression modifiers which helps to perform case-insensitive matching, a global matching between patterns, perform multiline matching with number of events. RE is a compact way of describing sets of strings which conform to a pattern, which analysis the sequence of patterns from the previous available database. The given pattern is compared with already stored text in database based on score function analysis.

set of characters that forms a meaningful pattern, mainly focus in pattern matching with strings. RE which performs search, matching and replacing patterns based on given input pattern . Regular Expression modifiers which helps to perform case-insensitive matching, a global matching between patterns, perform multiline matching with number of events. RE is a compact way of describing sets of strings which conform to a pattern, which analysis the sequence of patterns from the previous available database. The given pattern is compared with already stored text in database based on score function analysis.

\section{II.RELATED WORK}

\section{Rabin -Karp (RK)}

The Rabin-Karp string searching algorithm calculates the hash value of the input, for each character subsequence of pattern to be compared with given input. Hash value is used to identify match score between the patterns. If the match sore are equal, the algorithm will compare the pattern and repeated sequence of input. 
In this way, there is one alteration per single text subsequence, and character matching is required by any pre-processing of the pattern. For the pattern identical we need some additional possibilities in sequence, let's take a method once again to make among the common characters of the pattern with the concurrent character of the input message. RK algorithm requires to get quicker the testing of the idealness frequent model converts each and every string into a numeric value known as the hash value. i.e, we might have a hash ("AT") $=2$. RK uses the character equivalent. Thus, it will look at all we have to do is calculate seem for a substring with the ideal hash value. moreover, there are two agendas dissimilar or similar have a longer time for larger substrings.

Given a input string ' $b$ ', the case of string matching transaction with detecting whether a pattern ' $a$ ' occurs in ' $b$ ' and if ' $a$ ' does occur then recurring way in ' $b$ ' where ' $a$ ' occurs One of the most clear approaches in the direction selection ' $a$ ' with the first element of the string ' $b$ ' in which to locate ' $a$ '. If the first element of ' $a$ ' matches the first element of ' $b$ ', compare the second element of ' $a$ ' with the second element of ' $b$ '.

If match found proves similarly awaiting complete ' $a$ ' is detect to shift ' $a$ ' one position to the right and replicate comparison initiation from the starting element of ' $a$ '. This is a common randomized algorithm is used to execute in linear time in repeated states of affairs of practical interest.

\section{Boyce-Moore (BM)}

This Boyce-Moore algorithm initializes the input string thus individual searched in the text. This case its effective persevere crossways on number of sources. The BoyerMoore algorithm uses information's that are collected during the initializing method to unnecessary sections of the text, resulting in a lower. Commonly, the algorithm runs quickly as the pattern length increase the performance. The Boyer-Moore algorithm consider a pattern A against text B, a mismatch of text character B [i] $=\mathrm{p}$ with the corresponding pattern personality $\mathrm{A}[\mathrm{j}]$ is handled as follows: If $\mathrm{p}$ is not contained anywhere in A, then shift the pattern A completely past are [I]. Otherwise, shift A until an occurrence of characteristic pin A gets aligned with $\mathrm{B}[\mathrm{I}]$.

\section{DFA and NFA Automaton}

Choosing the interchange between DFAs and NFAs, number of systems make to use NFAs or extensions of NFAs. These systems tend to have an expressive pattern language where negations, Kleen closures, and temporal constraints are included. They are more emotional than regular expressions. These systems are impressive in the direction of quick processing over sequential event streams, where an event is hard, and contains excessive attributes. Our approach drives on a simpler trouble where events do not have excessive attributes, and this allows us to generate simpler algorithms.i.e, in accordance with the reputation of events is supported by initially finding all positive events and then pruning of the results that contain reputation of events in the fault temporal sequencing. Added as advantage, this algorithm searches for contradiction in-place. Theoretical derivations must be sufficiently labeled as hypothetical. Hypothetical performance will be calculated in the identical way as real performance. Members must be able to explain in the basis for the theoretical results and the underlying theory that generated them. Enable a single state of execution. Use a bit to represent the result to be derived is reached. Some transitions depends upon the bit values.

\section{III.PROPOSED SYSTEM}

Generic Pattern Matching follows the way of searching the string "XYZ" is given it searches first character X the $Y$ and as follows. On another case when two string are given, then the string with more number of character will be displayed as a result. i.e, when two strings, XYZ ABCD is given the count of first string is 3 and the count of second string is 4.then the string with more count "ABCD" will be displayed as the result since it has the greater count value compare to the other string.

\section{IV.ADVANTAGE}

The advantage of this approach is the ideality of avoiding the duplication of values so that it will be time and storage efficient.

- It also has a competitive value of Higher accuracy which gives it a added advantage comparing to other similar algorithms.

- Due to its closed approach this algorithm has a improved efficiency value.

\section{V.EXPERIMENTAL RESULTS}

Boyce-Moore algorithm is used for selected string patterns only. Average amount of commands executed against the pattern length. The total number of commands executed for a long time. Character decreases the running period. Rabin -Karp algorithm is used to running time executed worst performance. KMP algorithm is used to search the scanning existing character by character. This algorithm is used for best performance when compared these two it's very efficient. The temporal pattern search algorithm is efficient for design view. It is used to search for number of histories can take the rapid amount of time. It is used to skip the unnecessary events. This algorithm, when compared to the present algorithm it's very easy to find the pattern. It is easy to search the pattern and easy to maintain the search information.

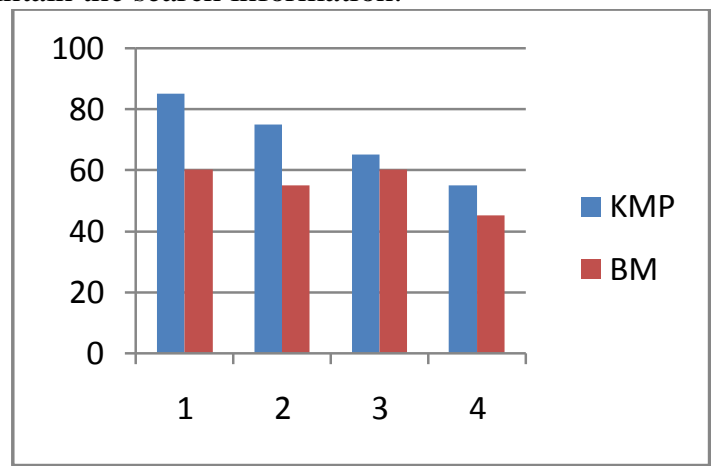

Fig:1. Comparison Between KMP and KM. 
This algorithm, when compared to the present algorithm it's very easy to find the pattern. It is easy to search the pattern and easy to maintain the search information. Here in this Fig.2, we compare the comparison between BM, RK and FA.

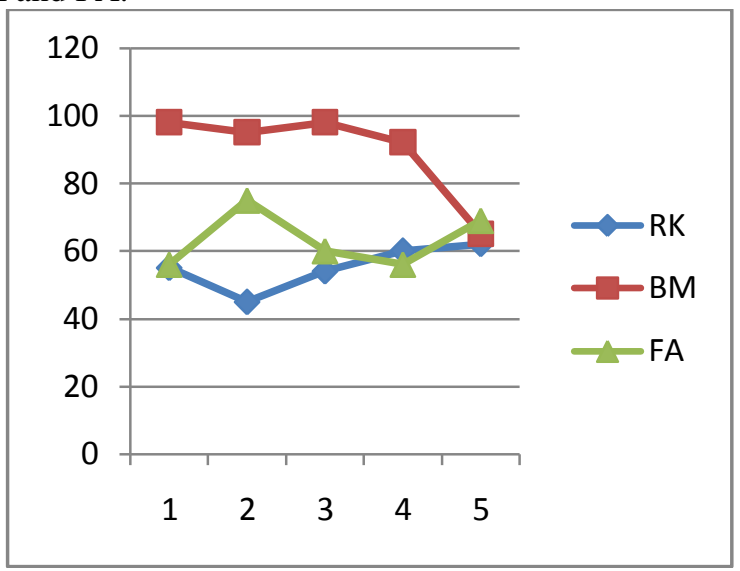

Fig:2. Comparison Between RK, BM and FA.

\section{VI.FUTURE ENHANCEMENT}

We present this paper on pattern matching to enhance the matching accuracy between the events. In this system since KMP and Quick sort is used thus it gives the exact result and value, It takes more time for generating the resulting event. Thus it is a Time complexity oriented system. As future work, it is interesting to consider the more complicated Time complexity for the events with different granularity in distinct processes. Moreover, we may exploit other attributes of events besides the accuracy and time adjustment.

\section{REFERENCES}

[1] R. Agrawal and R. Srikant. Mining sequential patterns. In P. S. Yuand A. L. P. Chen, editors, ICDE, pages 3-14. IEEE Computer Society, 1995 .

[2] C. Bettini, X. S. Wang, S. Jajodia, and J.-L. Lin. Discovering frequent event patterns with multiple granularities in time sequences. IEEE Trans.Knowl. Data Eng., 10(2):222-237, 1998.

[3] J. E. Cook and A. L. Wolf. Event-base detection of concurrency. InSIGSOFT FSE, pages 35-45, 1998.

[4] L. Ding, S. Chen, E. A. Rundensteiner, J. Tatemura, W.-P. Hsiung, and K. S. Candan. Runtime semantic query optimization for event stream processing. In ICDE, pages 676-685, 2008.

[5] X. Dong, A. Y. Halevy, and J. Madhavan. Reference reconciliation in complex information spaces. In SIGMOD Conference, pages 8596,2005.[8] D. R. Ferreira, D. Gillblad, and D. Gillblad. Discovering process models from unlabelled event logs. In BPM, pages 143-158, 2009.

[6] L. Gravano, P. G. Ipeirotis, N. Koudas, and D. Srivastava. Text joins in an rdbms for web data integration. In WWW, pages 90-101, 2003.

[7] G. Jeh and J. Widom. Simrank: a measure of structuralcontextsimilarity. In KDD, pages 538-543, 2002.

[8] J. Kang and J. F. Naughton. On schema matching with opaque column names and data values. In SIGMOD Conference, pages 205-216, 2003.

[9] D. Luckham. The power of events: An introduction to complex event processing in distributed enterprise systems. In RuleML, page 3, 2008.

[10] J. Madhavan, P. A. Bernstein, and E. Rahm. Generic schema matching with cupid. In VLDB, pages 49-58, 2001.

[11] S. Nejati, M. Sabetzadeh, M. Chechik, S. M. Easterbrook, and P. Zave.Matching and merging of statecharts specifications. In ICSE, pages 54-64, 2007.
[12] T. Pedersen, S. Patwardhan, and J. Michelizzi. Wordnet: : Similarity- measuring the relatedness of concepts. In AAAI, pages 1024-1025,2004.

[13] E. Rahm and P. A. Bernstein. A survey of approaches to automatic schema matching. VLDB J., 10(4):334-350, 2001. 